\title{
Head trauma in children-how to image?
}

\author{
Jean-François Chateil
}

(C) Springer-Verlag 2011

\begin{abstract}
Head trauma occurs very frequently in children, and is usually minor. There is ongoing debate about the use of imaging for initial diagnosis, follow-up, and for prediction of long-term functional morbidity. The pathophysiology of non-accidental injury is also controversial. This presentation provides an overview of the state-of-theart in imaging of head trauma in children.
\end{abstract}

Keywords Head trauma $\cdot$ Imaging $\cdot$ Child $\cdot$ CT $\cdot$ MRI

\section{Initial diagnosis}

Plain films are useful only in suspected non-accidental injury. Children with obvious skull fractures and/or Glasgow coma scale-score $<14$ need urgent $\mathrm{CT}$ of the head. The major benefit of $\mathrm{CT}$ is early identification of lesions that might otherwise be missed and, if untreated, increase the risk of morbidity and mortality. Clinicians frequently request CT for children following minor blunt head trauma. Unnecessary CT imaging, however, exposes the child needlessly to ionising radiation. CT is unlikely to show any clinically important intracranial lesions in children who do not exhibit at least one of the following: evidence of significant skull fracture, altered level of alertness, neurological deficit, persistent vomiting, presence of scalp haematoma, abnormal behaviour, or coagulopathy

Disclaimer Dr. Chateil has no financial interests, investigational or off-label uses to disclose.

J.-F. Chateil $(\bowtie)$

Service de Radiologie, Hôpital Pellegrin,

CHU Bordeaux,

33076 Bordeaux, France

e-mail: jean-francois.chateil@chu-bordeaux.fr
(National Emergency X-Radiography Utilization Study II, NEXUS II). Children younger than 2 years of age appear to have a higher risk of intracranial injury following minor head trauma. The negative prediction-factors in these young children are: normal mental status; no scalp haematoma, except frontal; no loss of consciousness, or loss of consciousness for less than $5 \mathrm{~s}$; non-severe injury mechanism; no palpable skull fracture; and unaltered behaviour according to their parents. These young patients can be difficult to assess clinically, and evidence recommends a more liberal approach to imaging with CT when the clinical findings are equivocal.

CT is useful to depict all haemorrhagic lesions: subdural and epidural haematoma, subarachnoid blood, and parenchymal contusion. Patients with moderate or severe head injury and intraparenchymal injuries are more likely to deteriorate; therefore head CT is done repeatedly in many of these cases. Deterioration may be due to developing brain oedema and vasospasm during treatment. MRI is used when CT findings do not explain the neurological symptoms. Transcranial Doppler US of the middle cerebral artery could also be performed to identify and follow vasospasm. Other cerebrovascular complications may include infarction and posttraumatic aneurysm; MRI is useful for these diagnoses. Arterial ischaemic stroke may occur as a result of trivial head or neck trauma (e.g. resulting from sports activity) causing carotid or vertebral arteries dissection. Head and neck angiographic CT or MRI is needed if this is suspected.

Associated cervical spine lesions are encountered mainly in infants with head injury resulting from road traffic accidents or falls from heights greater than $3 \mathrm{~m}$. In all other trauma cases, there is a low incidence $(0.2 \%)$ of cervical spine fractures. High-resolution $\mathrm{CT}$ with sagittal and coronal reconstructions should be the basis for cervical spinal clearance, and must be performed at the same time as the head CT. MRI is mandatory if neurological signs are present. 


\section{Long-term prognosis}

Higher levels of post-concussive symptoms after traumatic brain injury (TBI) in children are associated with road traffic accidents, loss of consciousness, neuroimaging abnormalities, and hospitalisation. Generalised brain volume loss has been well documented in moderate to severe head trauma, as has diffuse cerebral atrophy; white matter (mainly sub-cortical) may be affected more severely than grey matter. Diffuse axonal injury secondary to traumatic brain injury contributes to long-term functional morbidity. MRI with fluid-attenuated inversion recovery (FLAIR) and T2-weighted images is well known to depict such lesions during follow-up. T2, FLAIR, and susceptibility-weighted imaging (SWI) provide an accurate assessment of injury severity, and can detect lesions that are predictive of long-term outcome. The corpus callosum is particularly vulnerable, and callosal lesions have predictive value, along with, e.g., subcortical lesions. Significant global cortical thinning, compared to a cohort of normally developing children, has been reported following traumatic brain injury. Reduced cortical thickness is related to reported deficits in working memory. Reduced cerebellar white matter volume is also observed, and is consistent with evidence that the cerebellum and its related projection areas are highly vulnerable to fibre degeneration following trauma.

Several studies have tried to improve the sensitivity of MRI in these children. Diffusion-weighted imaging is useful for demonstrating subtle abnormalities. Apparent diffusion coefficient (ADC) values from peripheral regions are inversely correlated with cognitive function. The average whole-brain ADC value alone has a great ability to predict outcome, and seems to correctly predict outcome in the majority of cases. Diffusion fractional anisotropy (FA) measurements can show abnormalities despite an otherwise normal brain MRI. In several studies, higher FA in the corpus callosum was related to better functional outcome. Reduced size and microstructural changes in posterior callosal regions after brain injury suggest arrested development, decreased organisation, and disrupted myelination. FA of white matter correlates most strongly with neuropsychological outcome. FA in the frontal and supracallosal regions also correlates with executive functioning. Behaviour ratings (processing speed, working memory, executive deficit) correlate with supracallosal FA.

Magnetic resonance spectroscopy has been used to correlate the metabolic status of the corpus callosum with structural data (diffusion tensor imaging, DTI) and neuropsychological findings (cognitive function, bimanual coor- dination). Choline concentration was elevated during the post-acute but not chronic period. N-acetyl aspartate (a marker of neuronal/axonal integrity) was initially reduced and was positively correlated with DTI FA and most measures of cognitive function.

Attention deficit is common after paediatric TBI. A study examined brain activation patterns using functional MRI during a continuous performance task in a group of young children who had sustained TBI in early childhood, and in a group of age-matched controls with orthopaedic injuries. The TBI group demonstrated several areas of significantly greater activation relative to controls, including frontal and parietal regions. This could possibly be due to over-recruitment, compensating for damaged fibres and neurons.

Further technical development may allow earlier identification of predictors of chronic serious sequelae in the child following TBI, thereby facilitating rehabilitation, and also providing more accurate forensic evidence for any legal or insurance proceedings.

\section{Non-accidental head injuries}

CT remains the best tool in the emergency setting to detect subdural haematoma (SDH). SDH in infants most often result from non-accidental head injury (NAHI), which should be suspected in the absence of a plausible history of trauma, and by the presence of associated injuries. The existence of spontaneous SDH remains debated; it is very rare and should not be diagnosed unless NAHI has been positively excluded. The absence of traumatic features is not sufficient, and positive findings like macrocrania, arachnoidomegaly, or severe dehydration are necessary for the diagnosis of spontaneous SDH. In one previous retrospective study, cases in which a perpetrator confessed to violence toward the child were compared with cases in which there was no confession. There was no significant difference between the two groups for any of the variables studied: gender, mortality, fractures, retinal haemorrhage, ecchymoses, symptoms, and SDH pattern. Shaking was described as extremely violent $(100 \%)$ and was repeated (55\%) from two to 30 times (mean, 10) because it stopped the infant's crying (62.5\%). Impact was uncommon (24\%). No correlation was found between repeated shaking and SDH densities on CT. MRI is useful to search for associated brain lesions (T2*-weighted imaging or SWI are mandatory) but dating of different concentric layers of SDH is still very difficult. Epidural spinal haematoma can also be recognised, associated with NAHI. 